OPEN ACCESS

Edited by:

Tianjiao Ji,

Boston Children's Hospital and Harvard Medical School,

United States

Reviewed by:

Xingwang Zhang,

Jinan University, China

Hao Qin,

National Center for Nanoscience and

Technology (CAS), China

*Correspondence:

Wei He

weihe@cpu.edu.cn

†These authors have contributed equally to this work

Specialty section:

This article was submitted to

Supramolecular Chemistry,

a section of the journal

Frontiers in Chemistry

Received: 09 July 2020

Accepted: 28 July 2020

Published: 11 September 2020

Citation:

Zhang J, Teng C, Li C and He W (2020) Deliver Anti-inflammatory Drug Baicalein to Macrophages by Using a

Crystallization Strategy.

Front. Chem. 8:787.

doi: 10.3389/fchem.2020.00787

\section{Deliver Anti-inflammatory Drug Baicalein to Macrophages by Using a Crystallization Strategy}

\author{
Jianming Zhang ${ }^{1,2+}$, Chao Teng ${ }^{2 \dagger}$, Caolong $\mathrm{Li}^{3}$ and Wei He $\mathrm{He}^{4,2 *}$ \\ ${ }^{1}$ Shanghai Mental Health Center, Shanghai Jiao Tong University School of Medicine, Shanghai, China, ${ }^{2}$ School of Pharmacy, \\ China Pharmaceutical University, Nanjing, China, ${ }^{3}$ School of Science, China Pharmaceutical University, Nanjing, China, \\ ${ }^{4}$ Shanghai Skin Disease Hospital, Tongji University School of Medicine, Shanghai, China
}

Macrophages are potent to modulate inflammation via phenotypic switch and production of inflammatory factors. Baicalein (BCL) is frequently used to alleviate inflammation; however, its application is always hindered due to low solubility. Herein, BCL nanocrystals (BNRs) were prepared to improve its delivery to macrophages. The prepared BNRs have a diameter of $150 \mathrm{~nm}$ with a rod-like structure. The nanocrystals could be well-taken up by macrophages via the caveolar pathway and, therefore, promote the polarization switch from proinflammatory phenotype to anti-inflammatory macrophages and alleviate the inflammation via reducing production cytokine IL-12. In conclusion, the crystallization strategy is promising for the improvement of the solubility of $B C L$ and promotion of its anti-inflammatory activities.

Keywords: anti-inflammation, baicalein, macrophages, nanocrystals, low solubility

\section{INTRODUCTION}

Inflammation is an important life activity, protecting the body from infection with foreign organisms; however, chronic inflammation always results in exacerbation of numerous diseases such as cardiovascular diseases, arthritis, and inflammatory bowel disease (He W. et al., 2019). Macrophages play a central role in the regulation of inflammation via phenotypic switch and production of inflammatory factors (Leitinger and Schulman, 2013).

Baicalein (BCL), a major flavonoid of Scutellaria baicalensis, is a potent anti-inflammatory agent predominantly acting through associating with a variety of chemokines including IL-8, macrophage inflammatory protein (MIP)-1 $\beta$, and monocyte chemotactic protein (MCP)-2 and limiting their biological function (Li et al., 2000). Moreover, baicalein can polarize proinflammatory phenotype M1 to anti-inflammatory M2 and alleviate inflammatory response (Zhu et al., 2016). As a result, BCL has a promising potential to combat inflammation. However, its use is always limited due to low solubility in water (Zhang et al., 2009). Nanotechnology, such as liposomes, nanoemulsions, polymeric micelles, and nanocrystals, is potent to enhance the solubilization of poorly water-soluble active compounds, along with over 65 products approved for clinical use (Anselmo and Mitragotri, 2016; He H. et al., 2019; He et al., 2020; Yu et al., 2020). Of them, nanocrystal technology prepared by formulating drug particles into nanosized particles by top-down or bottom-up approaches is one of the most promising means to solve the solubility (Lu et al., 2019), owing to its advantages including its high drug loading, enhanced dissolution rate and saturation solubility, reproducibility of oral absorption, improved dose-bioavailability proportionality, and increased patient compliance (He et al., 2013, 2016; Lv et al., 2018; Zhou et al., 2018; Zhao et al., 2019). So far, over 15 
nanocrystal formulations such as Rapamune ${ }^{\circledR}$, Emend ${ }^{\circledR}$, Tricor ${ }^{\circledR}$, Megace $\mathrm{ES}^{\circledR}$, Avinza ${ }^{\circledR}$, Focalin $\mathrm{XR}^{\circledR}{ }^{\circledR}$, Ritalin ${ }^{\circledR}$, and Zanaflex Capsules ${ }^{\mathrm{TM}}$ have been approved. In particular, the formulation of nanocrystals has an extremely high drug-loading capacity that is markedly greater than that from conventional nanocarriers with the capacity of $<10 \%$ (He et al., 2013; Anselmo and Mitragotri, 2019). Accordingly, nanocrystal technology has a potential to address the solubility of BCL and improve its delivery.

In this study, BCL nanocrystals (BNRs) were prepared and characterized. Moreover, the uptake and internalization pathway in macrophages, phenotypic switch, and anti-inflammation in vitro were investigated, and finally, biocompatibility of BNRs was evaluated.

\section{MATERIALS AND METHODS}

\section{Materials}

Beta-lactoglobulin ( $\beta-\mathrm{LG})$, fluorescein isothiocyanate isomer I (FITC), rhodamine B isothiocyanate (RITC), 3-(4,5dimethylthiazol-2yl)-2,5-diphenyltetrazoliumbromide (MTT), and polyethylenimine (PEI, 408727, 25,000 Da) were provided by Sigma-Aldrich Co., Ltd. (St. Louis, MO, USA). Fetal bovine serum (FBS), Dulbecco's Modified Eagle Medium (DMEM), and trypsin were from Thermo Fisher Scientific, Inc. (Waltham, MA, USA). 4,6-Diamino-2-phenyl indole (DAPI) were brought from the Beyotime Institute of Biotechnology (Haimen, China). Nystain and methyl- $\beta$-cyclodextrin (M-CD) were supplied by Aladdin Co., Ltd. (Aladdin Co., Ltd., Shanghai, China). BCL was obtained from Chengdu Pufei De Biotech Co., Ltd. (Chengdu, China). Antibodies of CD206 and INOS were obtained from Proteintech Co., Ltd. (Rosemont, USA). Cy5 was obtained from Solarbio Science \& Technology Co., Ltd. (Shanghai, China). Alexa Fluor ${ }^{\circledR}$ 488-Cave-1/F-actin/CTB was from Abcam Trading Co., Ltd. (Shanghai, China). ELISA kits of mouse interferon- $\alpha$ (INF- $\alpha$ ) and interleukin-12 (IL-12) were purchased from Enzyme-linked Biotechnology Co. Ltd. (Shanghai, China).

\section{Cell Cultures and Animals}

RAW 264.7 cells were cultured in DMEM containing 10\% FBS and $1 \%$ penicillin/streptomycin at $37^{\circ} \mathrm{C}, 5 \% \mathrm{CO}_{2}$, and $100 \%$ humidity and were split when confluent. Before use, the cells were polarized into M1 phenotype by incubation with lipopolysaccharides $(100 \mathrm{ng} / \mathrm{mL})$ and interferon- $\gamma(20 \mathrm{ng} / \mathrm{mL})$ for $24 \mathrm{~h}$.

The animals used in all experiments received care in compliance with the Principles of Laboratory Animal Care and the Guide for the Care and Use of Laboratory Animals. Animal experiments followed a protocol approved by the China Pharmaceutical University Institutional Animal Care and Use Committee.

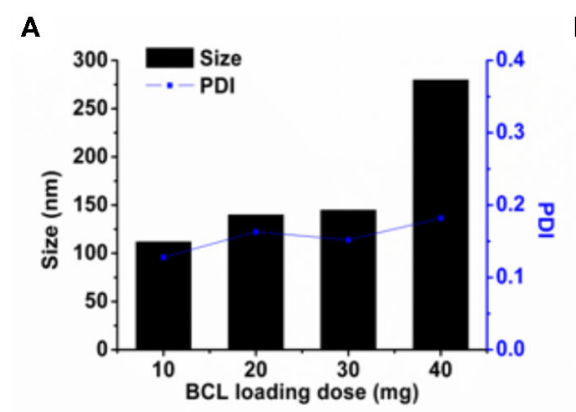

B

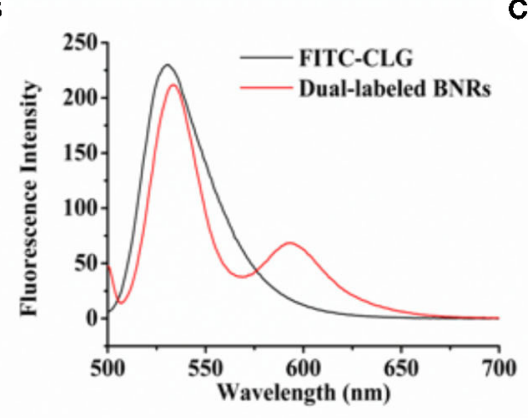

E

D
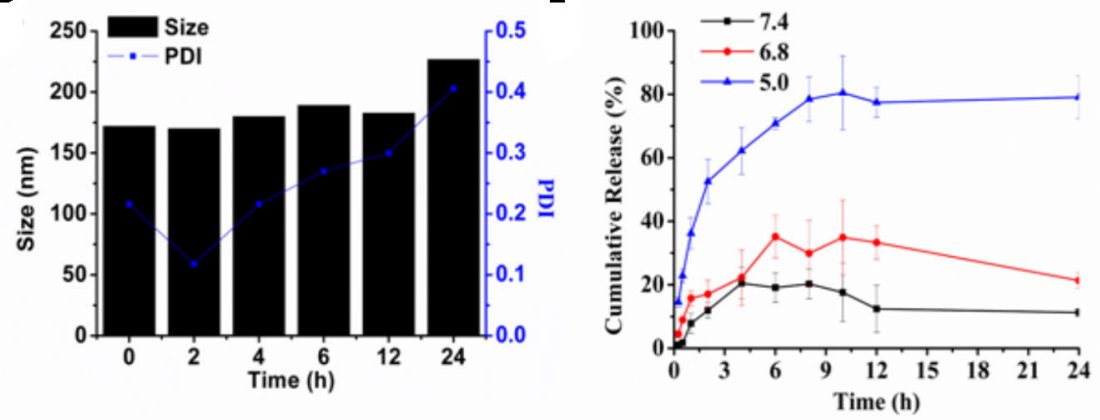

C

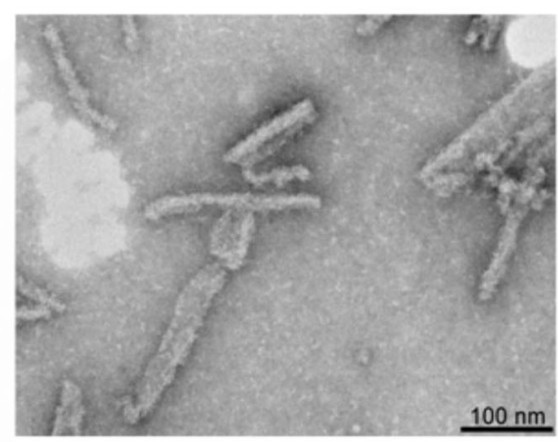

FIGURE 1 | Preparation and characterization. (A) Effect of baicalein loading on the particle size of BNRs. (B) Fluorescence emission spectra for FRET assay. FITC and RITC were used to label CLG and the drug crystals, respectively. Dual-labeled BNRs were prepared by coating the RITC-labeled drug crystals with FITC-CLG. Excitation wavelength: $492 \mathrm{~nm}$. (C) TEM image of optimized formulation (scale bar $=100 \mathrm{~nm}$ ). (D) Particle-size change of BNRs in $10 \%$ serum at $37^{\circ} \mathrm{C}$ for $12 \mathrm{~h}$. (E) in vitro release of baicalein from BNRs in buffer solution at $\mathrm{pH}$ of $5.0,6.8$, and 7.4 at $37^{\circ} \mathrm{C}$ for $24 \mathrm{~h}$. 


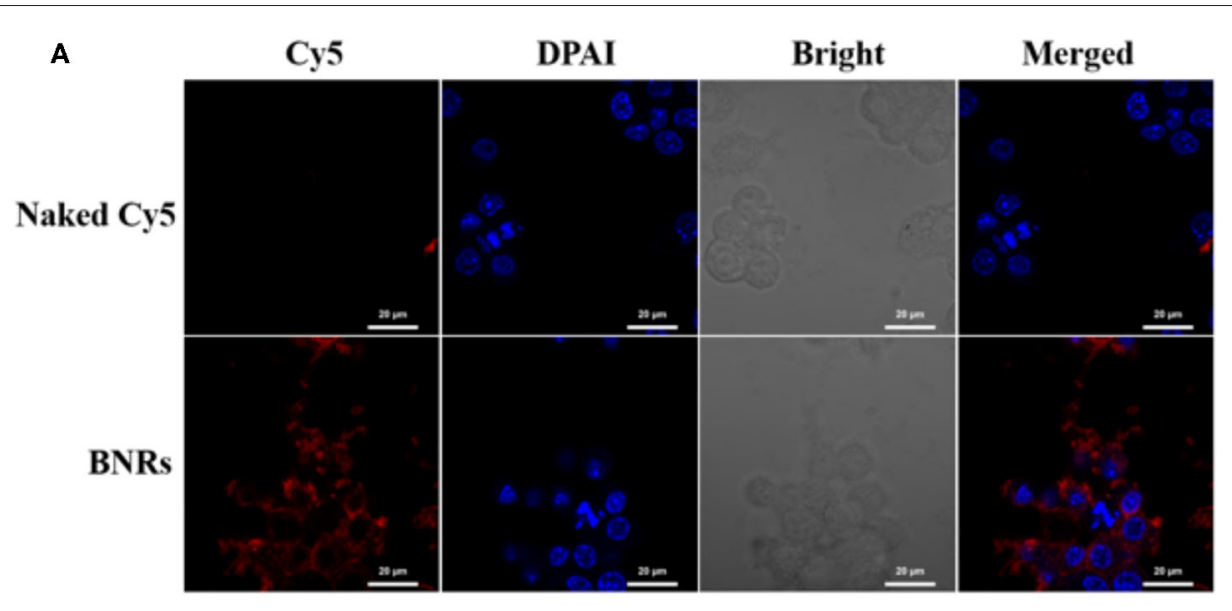

B

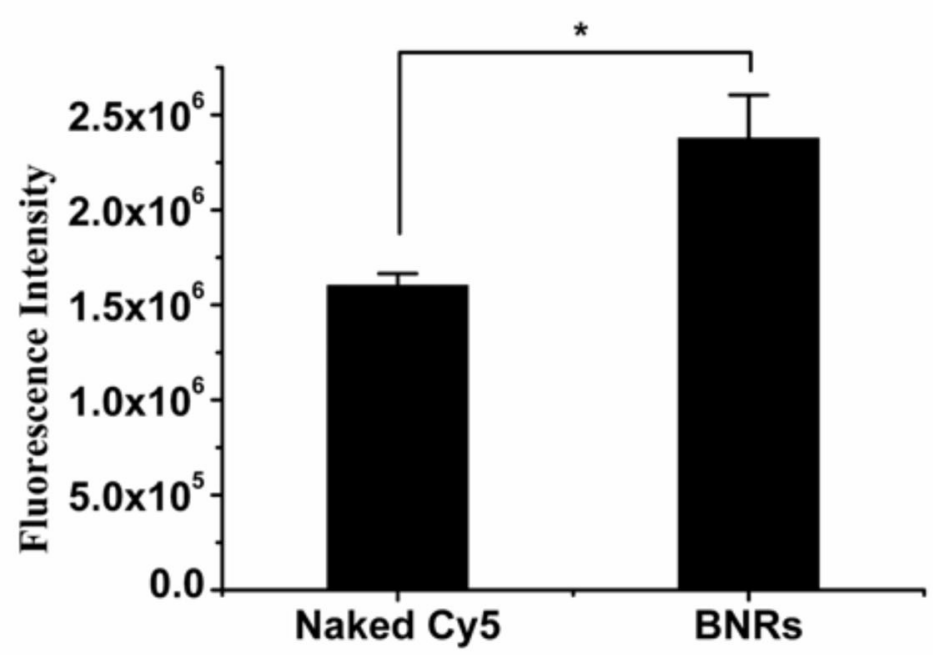

FIGURE 2 | Uptake in macrophages. Uptake of Cy5-labeled BNRs in RAW 264.7 cells was tested by (A) CLSM and (B) flow cytometry, following incubation at a dye concentration of $100 \mathrm{nM}$ at $37^{\circ} \mathrm{C}$ for $4 \mathrm{~h}$ (mean $\pm \mathrm{SD}, n=3$, ${ }^{*} P<0.05$ ). The scale bar is $10 \mu \mathrm{m}$.

\section{Preparation and Characterization of Baicalein Nanocrystals}

The BCL nanocrystals (BNRs) were prepared by the precipitation-ultrasonication method with a cationic betalactoglobulin (CLG) as a stabilizer (He et al., 2013; Xin et al., 2019). Briefly, BCL dissolved in $1 \mathrm{~mL}$ dimethyl sulfoxide (DMSO) was mixed with $10 \mathrm{~mL}$ CLG solution $(1 \mathrm{mg} / \mathrm{mL})$ under stirring and treated with an ultrasonic probe $(20-25 \mathrm{kHz}$, Scientz Biotechnology Co., Ltd., Ningbo, China) at $300 \mathrm{~W}$ for $10 \mathrm{~min}$ in an ice bath. Dye-labeled nanoparticles were prepared by a similar method except for dissolving the dye and the drug in DMSO together in advance for the mixing.

Serum stability of nanoparticles was performed by incubation in $\mathrm{PBS}$ containing $10 \% \mathrm{FBS}$ at $37^{\circ} \mathrm{C}$. At specific time points, the particle size was tested by using a 90Plus Particle Size Analyzer (Brookhaven Instruments, Holtsville, NY) at $25^{\circ} \mathrm{C}$.

The nanoparticles' shape was tested by a transmission electron microscope (TEM, JEM-1230, Tokyo, Japan) under an acceleration voltage of $200 \mathrm{kV}$. In brief, one drop of diluted sample was placed onto the copper mesh, and then the mesh was dried, stained with $2 \%(\mathrm{w} / \mathrm{w})$ phosphotungstic acid for $30 \mathrm{~s}$, and finally dried at $25^{\circ} \mathrm{C}$ for $5 \mathrm{~min}$.

The coating of the stabilizer on the nanoparticles was studied by fluorescence resonance energy transfer (FRET) in a fluorescence spectrometer (SHIMADZU RF-5301PC, Japan), in which FITC and RITC were utilized as the energy donors and acceptors. The scanning was performed at an excitation wavelength of $492 \mathrm{~nm}$. The split width was $5 \mathrm{~nm}$ for the excitation and $15 \mathrm{~nm}$ for the emission.

\section{In vitro Drug Release}

The drug release was investigated by a dialysis method. In brief, the samples were added into a dialysis bag $(3,500 \mathrm{Da})$ and immersed in a shaking incubator (SHA-C, Jintan, China) with a speed of $100 \mathrm{rpm} / \mathrm{min}$ at $37^{\circ} \mathrm{C}$. At pre-determined time intervals, samples were collected and purified with a $0.2 \mu \mathrm{m}$ 
A

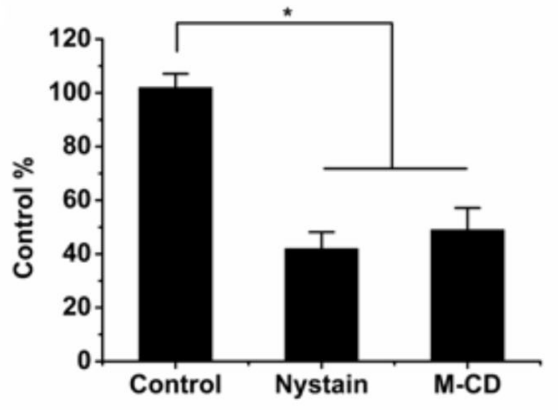

B
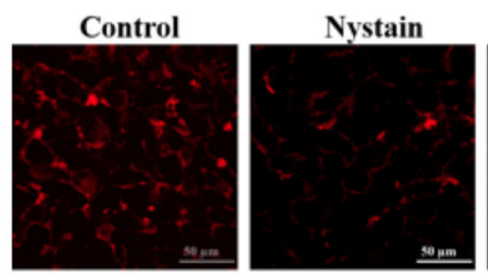

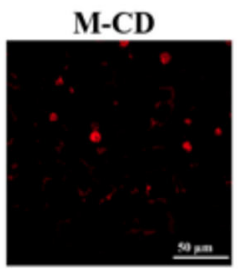

c

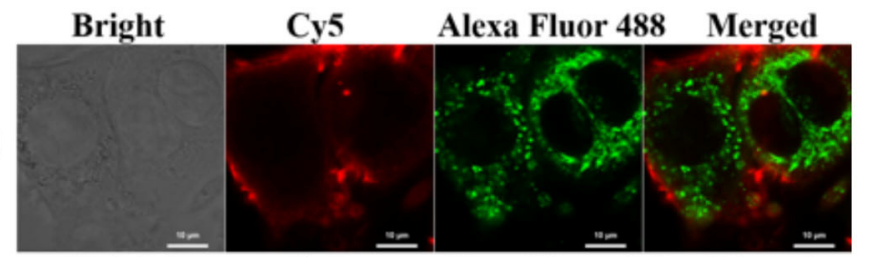

Cave-1

Bright

Cy5

Alexa Fluor 488 Merged

CTB

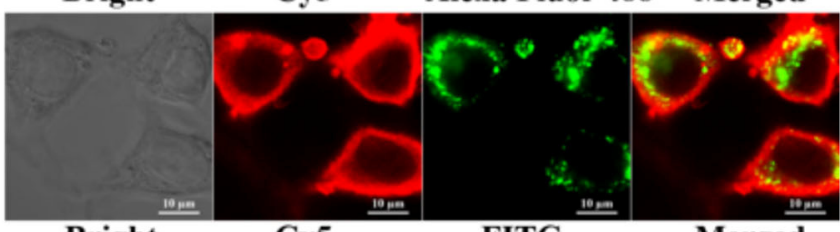

Bright
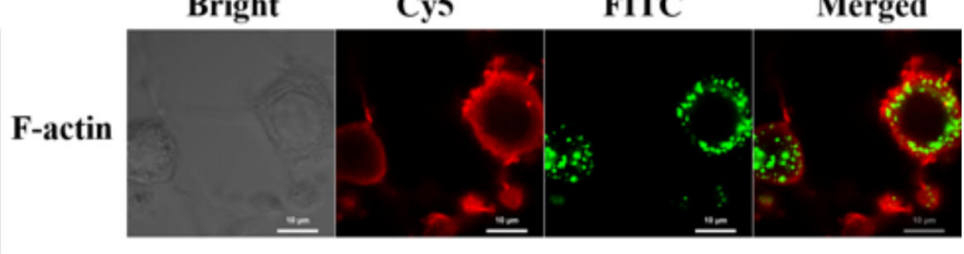

FIGURE 3 | Internalization pathway. (A,B) Cellular uptake of Cy5-labeled BNRs in RAW 264.7 cells pre-treated with different inhibitors for $0.5 \mathrm{~h}$ was determined by (A) flow cytometry (mean $\pm \mathrm{SD}, n=3,{ }^{*} P<0.01$ ) and (B) CLSM observation after a $4 \mathrm{~h}$ incubation at $37^{\circ} \mathrm{C}(\mathrm{Scale}$ bar, $50 \mu \mathrm{m})$. (C) Colocalization of Cy5-labeled BNRs with caveolae-trafficking proteins, Alexa Fluor 488-labeled CTB (green), Alexa Fluor 488-labeled Cave-1 (green), and FITC-labeled F-actin (green). The scale bar is $10 \mu \mathrm{m}$.

filter. The drug content was assayed in a high-performance liquid chromatography system equipped with an ultraviolet detector (SHIMAZU LC-10AT, Kyoto, Japan). The separation was performed on the ODS C18 column $(250 \mathrm{~mm} \times 4.6 \mathrm{~mm}$, Diamonsil, Beijing, China) at $30^{\circ} \mathrm{C}$ at $276 \mathrm{~nm}$. The mobile phase consisted of methanol and $0.05 \%$ phosphoric acid $(70 / 30, \mathrm{v} / \mathrm{v})$ and was pumped at a flow rate of $1 \mathrm{~mL} / \mathrm{min}$ (Teng et al., 2020).

\section{Cell Experiments}

Cells cultured on the 6-well plates $\left(1 \times 10^{5}\right.$ cells/well $)$ were incubated with the samples for $4 \mathrm{~h}$ and then were subjected to determination by flow cytometry (BD FACS Calibur, San Jose, CA, USA) and observation by using confocal laser scanning microscopy (CLSM, LSM700, Carl Zeiss, Germany). To study the internalization pathway, the cells were pre-incubated with inhibitors, nystatin $(10 \mathrm{mM})$, or M-CD $(2.5 \mathrm{mM})$, for $0.5 \mathrm{~h}$. The colocalization was examined by confocal microscopy after incubation with the nanoparticles and staining with Alexa Fluor ${ }^{\circledR} 488$-Cave-1, -F-actin, or -CTB for $3 \mathrm{~h}$.

The polarization of RAW 264.7 cells were assayed by CLSM and flow cytometry. Briefly, the cells $\left(1 \times 10^{5}\right.$ cells/well $)$ were cultured with the preparations for $12 \mathrm{~h}$, incubated with a primary and secondary antibody for 2 and $24 \mathrm{~h}$ at $4{ }^{\circ} \mathrm{C}$, respectively, and stained with DAPI for $15 \mathrm{~min}$. The anti-inflammation in vitro was tested by detecting IL-12 with ELISA kits after a $12 \mathrm{~h}$ incubation with various preparations. Cell viability was studied by MTT assay after incubation with preparations for $24 \mathrm{~h}$.

\section{Biocompatibility Study}

Hemolytic activity was studied by incubating the formulations with erythrocytes. In brief, erythrocytes were collected from mouse blood by centrifugation at $1,500 \times \mathrm{g}$ for $15 \mathrm{~min}$, wash, and resuspension with PBS. Then, CLG or PEI was incubated with the erythrocytes at $37^{\circ} \mathrm{C}$ for $1 \mathrm{~h}$, followed by centrifugation at $1,500 \times$ $\mathrm{g}$ for $15 \mathrm{~min}$, collection of the supernatant, and comparison of hemolytic activity.

The immunogenicity was assessed by detecting IL-12 and INF- $\alpha$ in the plasma with ELISA kits at pre-determined time intervals after intravenous injection of various preparations at specific doses according to the body weight of mouse.

\section{Statistical Analysis}

One-way analysis of variance was performed to assess the statistical significance of the differences between samples. The results are expressed as the mean \pm standard deviation (SD). $P<0.05$ indicated significant differences.

\section{RESULTS AND DISCUSSION}

\section{Preparation and Characterization of BNRs}

CLG is a safe biopolymer and potent to stabilize drug crystals (He et al., 2013; Teng et al., 2020). Herein, BNRs were prepared via a precipitation-ultrasonication method by using CLG as a stabilizer. First, the influence of drug loading on the diameter of BNRs was investigated. As displayed in Figure 1A, increasing the drug loading from 10 to $40 \mathrm{mg}$ in a $10 \mathrm{~mL}$ CLG solution containing $10 \mathrm{mg}$ CLG resulted in an increase of particle size from 125 to $280 \mathrm{~nm}$. Nonetheless, the diameter was $<150 \mathrm{~nm}$ as the added drug was $<30 \mathrm{mg}$, and all PDI values are smaller than 0.3 , indicating a homogeneous dispersion with narrow size 


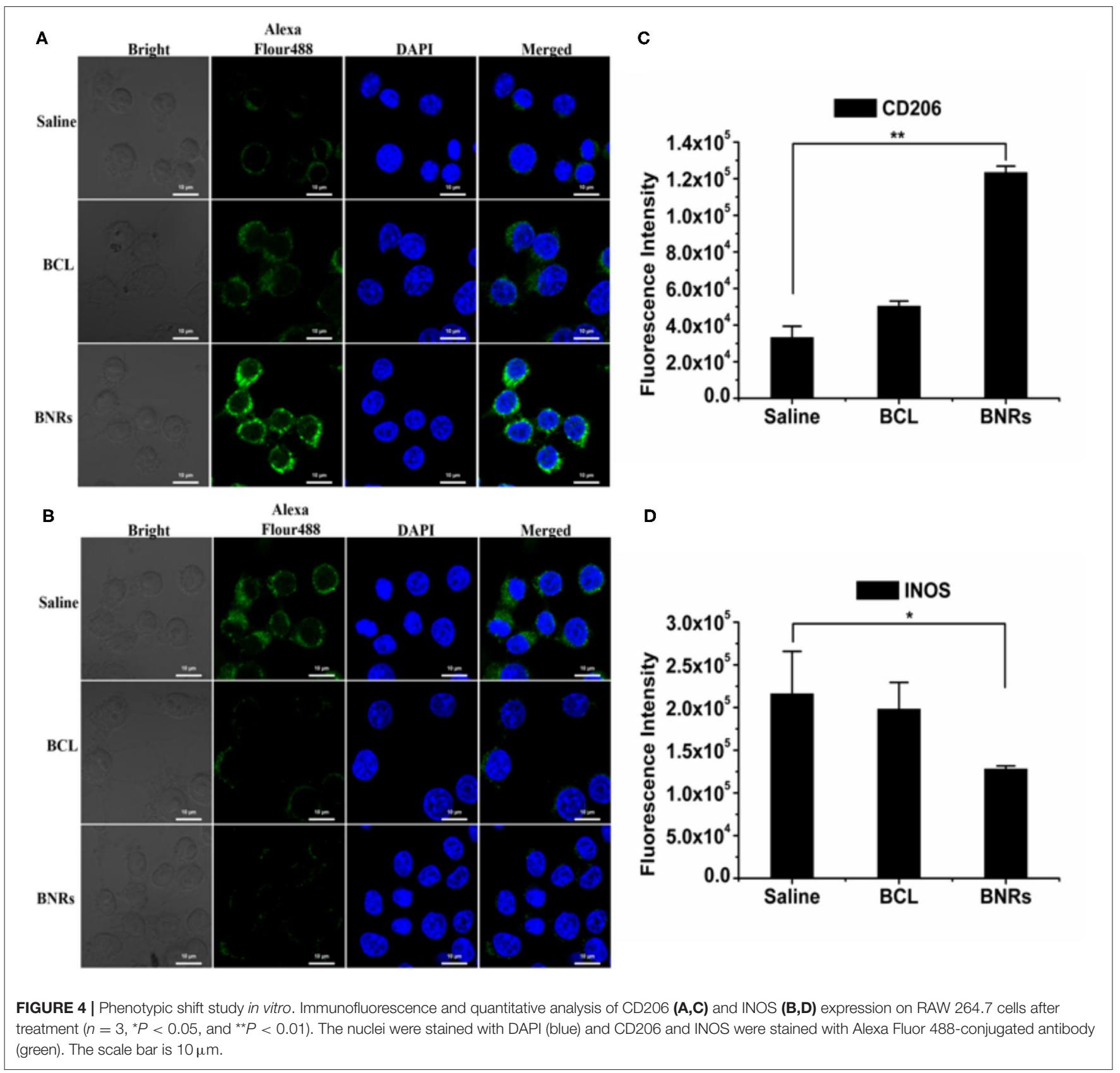

distribution of the nanoparticles. The results demonstrated that the stabilizer CLG is potent to stabilize the drug nanocrystals. To identify the interaction between the drug crystals and CLG, FRET was utilized, in which FITC and RITC were used as the energy donors and acceptors, respectively. FRET effect is displayed via the reduction fluorescence intensity of donor at $520 \mathrm{~nm}$ with increased fluorescence of the acceptor at $590 \mathrm{~nm}$ (Figure 1B), an indicator of the interplay between the stabilizer and the crystals. TEM examination demonstrated that BNRs has a rod shape and diameter of $100-150 \mathrm{~nm}$ in length (Figure 1C). The test of serum stability performed via incubation in $10 \%$ FBS displayed little alternation in the diameter of the nanoparticles at $12 \mathrm{~h}$ post-incubation and, as a result, demonstrated the potential stability in physiological conditions (Figure 1D).

The drug release profiles were studied at three $\mathrm{pH}$ conditions, $\mathrm{pH} 5,6.8$, and 7.4, through a dialysis method. The drug release at pHs of 6.8 and 7.4 was $<40$ and $20 \%$ in a 24 h period, respectively, whereas the release at $\mathrm{pH} 5$ was up to $80 \%$ (Figure 1E). The results indicated that the drug release from BNRs was $\mathrm{pH}$ dependent due to that BCL is a weak basic drug that has higher solubility in low $\mathrm{pH}$ conditions. 


\section{Cellular Uptake via Caveolar Pathway}

First, the uptake in macrophages was evaluated. After a $4 \mathrm{~h}$ incubation, strong red fluorescent spots around the nucleic were displayed (Figure 2A), confirmed by the determination by flow cytometry (Figure 2B). Second, previous reports demonstrated that rod-like particles with a diameter of $<200 \mathrm{~nm}$ in length always obtained cellular entry via the caveolar pathway, bypassing the endo-lysosomal systems (Xin et al., 2017, 2018, 2019). As depicted in Figure 3A, the internalization of BNRs decreased by $\sim 60 \%$ in the cells pre-treated with inhibitors of caveolar pathway, nystatin, or M-CD, compared with the control pre-treated with saline. The reduced uptake was verified by the examination by fluorescence microscopy (Figure 3B). In order to confirm the participation of the caveolar pathway in the uptake, co-localization of Cy5-labeled BNRs with caveolae-trafficking proteins, Cav-1, CTB, and F-actin, was studied using CLSM.

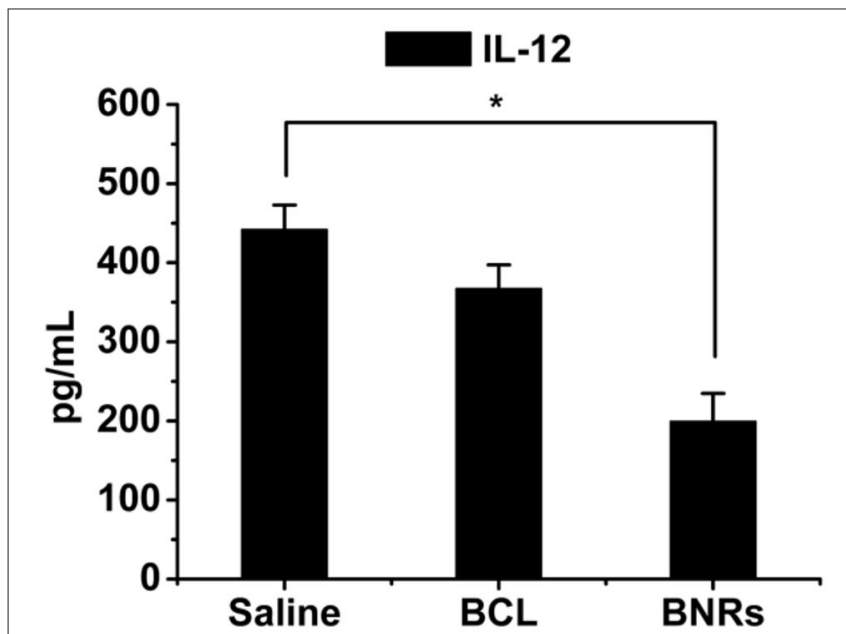

FIGURE 5 | Anti-inflammation in vitro. Quantitative analysis of IL-12 expression on RAW 264.7 cells after treatment for $12 \mathrm{~h}\left(n=3,{ }^{*} P<0.05\right)$.
Yellow fluorescent spots that appeared in the merged pictures demonstrated the colocalization of the nanoparticles with the three proteins (Figure 3C). These results indicated that $150 \mathrm{~nm}$ BNRs entered cells mainly via the caveolar pathway.

\section{Enhanced Phenotype Switch From M1 to M2 and Anti-inflammation}

$\mathrm{BCL}$ is potent to alleviate anti-inflammation effects. In this study, we hypothesized that BNRs allowed for reduced inflammation activities via promoting the phenotypic switch of macrophages from proinflammatory phenotype (M1) to antiinflammatory phenotype (M2). To characterize the polarization of macrophages, two markers, CD206 and INOS, that highly express on phenotypic $\mathrm{M} 2$ and $\mathrm{M} 1$, respectively (He W. et al., 2019), were detected by immunofluorescence assay. Treatment with free BCL or BNRs upregulated CD206 compared with saline (Figure 4A), confirmed by the quantified results assayed by flow cytometry (Figure 4B). Importantly, BNRs enabled an $\sim 2$-fold increase in the expression of CD206 over free BCL. Also, treatment with BNRs reduced the expression of INOS significantly (Figures 4C,D). Next, the anti-inflammatory effect was assessed in macrophages after incubation with various formulations. As expected, dosing BNRs significantly reduced the secretion of inflammatory factor, IL-12, along with a $>50 \%$ decrease with that from BCL (Figure 5). The results demonstrated that BNRs polarized M1 into M2 with high efficacy and leased the inflammation effectively.

The enhanced anti-inflammation effect in vitro resulted from the benefits of BNRs, high drug-loading capacity, and sustained drug release. To react to the surrounding situations, macrophages tend to alter their polarization, implying that the polarization may be changed once the drug's simulation disappears $(\mathrm{He}$ W. et al., 2019). As is well-known, most of drug delivery systems (DDS) release their drugs rapidly in cells after uptake due to the decomposition by the endo-lysosomes ( $\mathrm{He}$ et al., 2020), always discounting the inflammatory activity of drugs. Previous reports indicated that the drug crystals in cells could
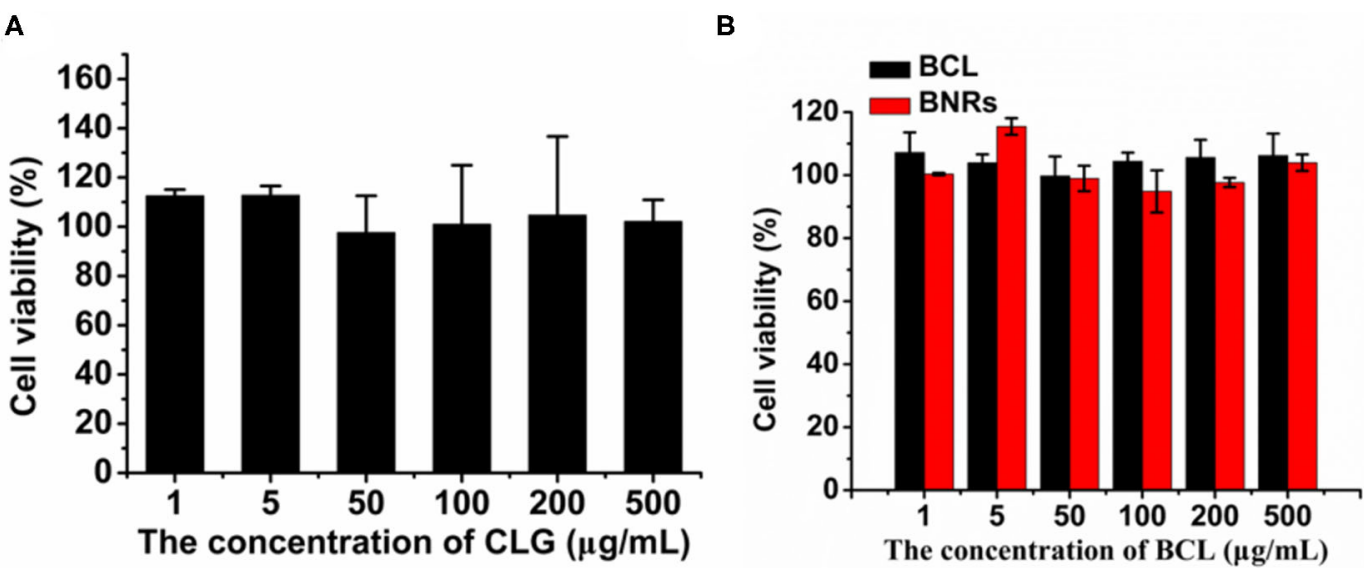

FIGURE 6 | Cell viability. Cytotoxicity of (A) stabilizer CLG and (B) formulations to RAW 264.7 cells post $48 \mathrm{~h}$ incubation. 
A

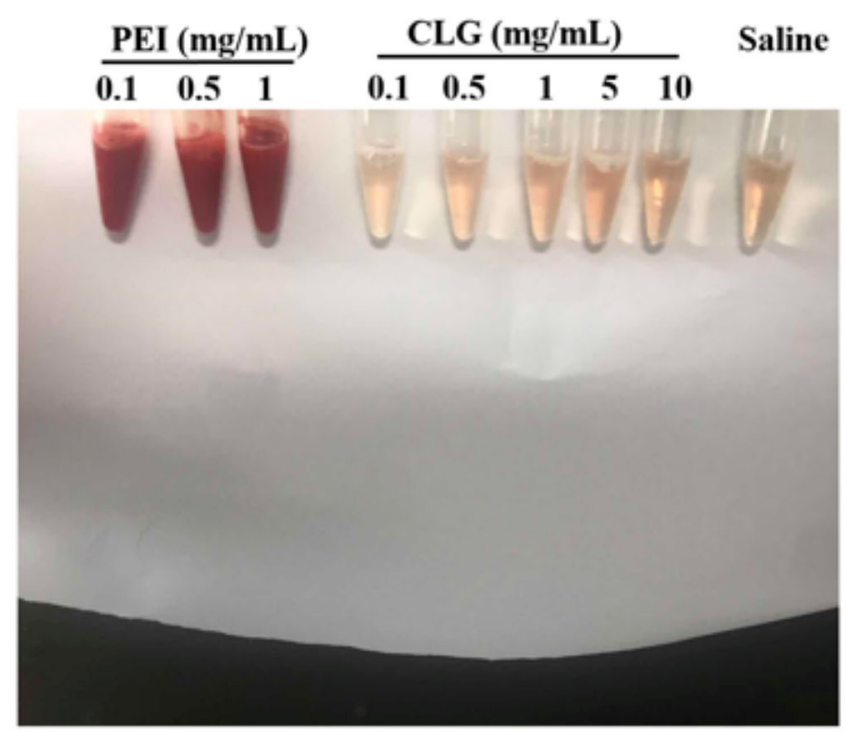

B

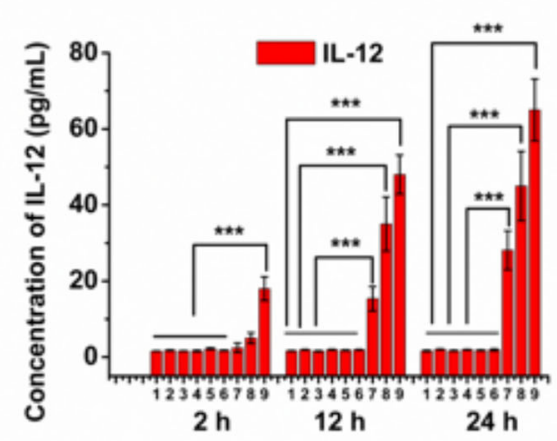

C

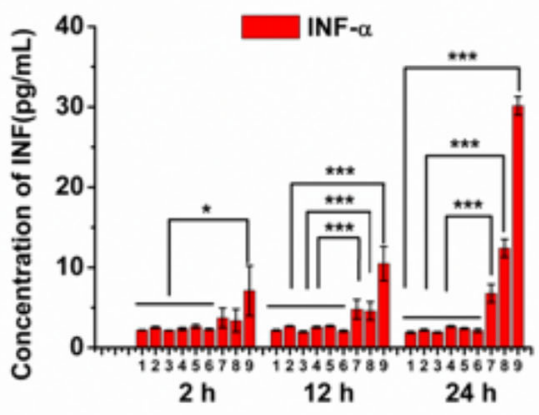

FIGURE 7 | Biocompatibility. (A) Hemolytic activity of CLG $(0.1-10 \mathrm{mg} / \mathrm{mL})$. A cationic vector, PEI, was used as a positive control. Inflammatory factors, (B) IL-12, and (C) INF- $\alpha$, in the mouse plasma after injection of CLG or PEl for 2, 12, and $24 \mathrm{~h}$, respectively. The formulations and administration doses based on the body weight are as follows: 1, saline; 2, CLG, $1 \mathrm{mg} / \mathrm{kg} ; 3, \mathrm{CLG}, 5 \mathrm{mg} / \mathrm{kg} ; 4, \mathrm{CLG}, 10 \mathrm{mg} / \mathrm{kg} ; 5, \mathrm{CLG}, 50 \mathrm{mg} / \mathrm{kg} ; 6, \mathrm{CLG}, 100 \mathrm{mg} / \mathrm{kg} ; 7, \mathrm{PEl}, 1 \mathrm{mg} / \mathrm{kg} ; 8, \mathrm{PEl}, 2.5 \mathrm{mg} / \mathrm{kg} ; 9, \mathrm{PEl}$, $5 \mathrm{mg} / \mathrm{kg} \cdot n=3,{ }^{*} P<0.05$ and ${ }^{\star \star \star} P<0.001$.

keep their integrity up to 7-10 h ( $\mathrm{Lu}$ et al., 2017; Qi et al., 2019). In this study, the BNRs with a payload capacity of 7-10-fold increase over conventional DDS entered cells via bypassing the endo-lysosomes and, as a result, maintained their integrity in the cytosol. These profiles allowed BNRs to sustain release of BCL over time after uptake. Indeed, as displayed in Figure 1E, BNRs released the drug in a slow pattern. Overall, the nanocrystal approach is promising to improve the efficacy of anti-inflammatory drugs against inflammatory diseases such as myocarditis, atherosclerosis, pulmonary hypertension, stroke, and cardiac disease.

\section{Biocompatibility Study}

First, the cell viability of the stabilizer CLG used in the formulations and BNRs was determined by the MTT assay. CLG and BNRs have little cytotoxicity to macrophages in the tested concentrations (Figure 6). Then, hemolytic activity was investigated by using PEI, a cationic vector, as positive control. Incubation with PEI resulted in profound hemolysis. In contrast, treatment with CLG at pre-determined concentrations showed ignored hemolytic toxicity (Figure 7A). In addition, the immunogenicity was evaluated by determination of inflammatory factors, IL-12 and INF- $\alpha$, in the plasma after intravenous injection of CLG or PEI at specific doses. The levels of the two factors from the groups dosed with PEI went up markedly at 12 or $24 \mathrm{~h}$ post-injection compared with that from the saline-treated group (Figures $7 \mathbf{B}, \mathbf{C}$ ), whereas the injection of CLG did not increase the concentration of the factors. The results indicated that CLG is safe for intravenous injection.

\section{CONCLUSIONS}

In this study, BCL nanocrystals were prepared and characterized. The nanocrystals have a diameter of $\sim 150 \mathrm{~nm}$ with a rod-like structure. Via internalization through the caveolar pathway, the drug nanocrystals are potent to promote the phenotypic switch from proinflammatory M1 to anti-inflammatory M2 and, as a result, alleviate the inflammation activity in vitro. In conclusion, crystallization is a promising strategy to improve the activity of insoluble anti-inflammatory agents.

\section{DATA AVAILABILITY STATEMENT}

All datasets presented in this study are included in the article.

\section{ETHICS STATEMENT}

The animal study was reviewed and approved by The China Pharmaceutical University Institutional Animal Care and Use 
Committee. Written informed consent was obtained from the owners for the participation of their animals in this study.

\section{AUTHOR CONTRIBUTIONS}

WH conceived and designed the research work. JZ, CT, and CL performed the experiments. All of the authors discussed the results and commented on the manuscript. All of the authors have read and approved the final manuscript.

\section{REFERENCES}

Anselmo, A. C., and Mitragotri, S. (2016). Nanoparticles in the clinic. Bioeng. Transl. Med. 1, 10-29. doi: 10.1002/btm2.10003

Anselmo, A. C., and Mitragotri, S. (2019). Nanoparticles in the clinic: an update. Bioeng. Transl. Med. 4:e10143. doi: 10.1002/btm2.10143

He, H., Lu, Y., Qi, J., Zhu, Q., Chen, Z., and Wu, W. (2019). Adapting liposomes for oral drug delivery. Acta Pharm. Sin. B 1, 36-48. doi: 10.1016/j.apsb.2018.06.005

He, W., Lu, Y., Qi, J., Chen, L., Hu, F., and Wu, W. (2013). Food proteins as novel nanosuspension stabilizers for poorly water-soluble drugs. Int. J. Pharm. 441, 269-278. doi: 10.1016/j.ijpharm.2012.11.033

He, W., Lv, Y., Xiao, Q., Ye, L., Cai, B., Qin, C., et al. (2016). Denatured protein stabilized drug nanoparticles: tunable drug state and penetration across the intestinal barrier. J. Mater. Chem. B 5, 1081-1097. doi: 10.1039/C6TB02577C

He, W., Kapate, N., Shields C. W. IV, and Mitragotri, S. (2019). Drug delivery to macrophages: a review of targeting drugs and drug carriers to macrophages for inflammatory diseases. Adv. Drug Deliv. Rev. doi: 10.1016/j.addr.2019.12.001. [Epub ahead of print].

He, W., Xing, X., Wang, X., Wu, D., Wu, W., Guo, J., et al. (2020). Nanocarrier-mediated cytosolic delivery of biopharmaceuticals. Adv. Funct. Mater. doi: 10.1002/adfm.201910566. [Epub ahead of print].

Leitinger, N., and Schulman, I. G. (2013). Phenotypic polarization of macrophages in atherosclerosis. Arterioscler. Thromb. Vasc. Biol. 33, 1120-1126. doi: 10.1161/ATVBAHA.112.300173

Li, B. Q., Fu, T., Gong, W. H., Dunlop, N., Kung, H., Yan, Y., et al. (2000). The flavonoid baicalin exhibits anti-inflammatory activity by binding to chemokines. Immunopharmacology 49, 295-306. doi: 10.1016/S0162-3109(00)00244-7

Lu, Y., Lv, Y., and Li, T. (2019). Hybrid drug nanocrystals. Adv. Drug Deliv. Rev. 143, 115-133. doi: 10.1016/j.addr.2019.06.006

Lu, Y., Qi, J., Dong, X., Zhao, W., and Wu, W. (2017). The in vivo fate of nanocrystals. Drug Discov. Today 22, 744-750. doi: 10.1016/j.drudis.2017.01.003

Lv, Y., Xu, C., Zhao, X., Lin, C., Yang, X., Xin, X., et al. (2018). Nanoplatform assembled from a CD44-targeted prodrug and smart liposomes for dual targeting of tumor microenvironment and cancer cells. ACS Nano 12, 1519-1536. doi: 10.1021/acsnano.7b08051

Qi, J., Hu, X., Dong, X., Lu, Y., Lu, H., Zhao, W., et al. (2019). Towards more accurate bioimaging of drug nanocarriers: turning aggregationcaused quenching into a useful tool. Adv. Drug Deliv. Rev. 143, 206-225. doi: 10.1016/j.addr.2019.05.009

\section{FUNDING}

This study was supported by the National Natural Science Foundation of China (No. 81872823), the Double FirstClass (CPU2018PZQ13) of the CPU, the Shanghai Science and Technology Committee (19430741500), and the Key Laboratory of Modern Chinese Medicine Preparation of Ministry of Education of Jiangxi University of traditional Chinese Medicine.

Teng, C., Lin, C., Huang, F., Xing, X., Chen, S., Ye, L., et al. (2020). Intracellular codelivery of anti-inflammatory drug and anti-miR 155 to treat inflammatory disease. Acta Pharm. Sin. B. doi: 10.1016/j.apsb.2020.06.005. [Epub ahead of print].

Xin, X., Du, X., Xiao, Q., Azevedo, H. S., He, W., and Yin, L. (2019). Drug nanorodmediated intracellular delivery of microRNA-101 for self-sensitization via autophagy inhibition. Nano Micro Lett. 11:82. doi: 10.1007/s40820-019-0310-0

Xin, X., Pei, X., Yang, X., Lv, Y., Zhang, L., He, W., et al. (2017). Rod-shaped active drug particles enable efficient and safe gene delivery. Adv. Sci. 4:1700324. doi: 10.1002/advs.201700324

Xin, X., Teng, C., Du, X., Lv, Y., Xiao, Q., Wu, Y., et al. (2018). Drug-deliveringdrug platform-mediated potent protein therapeutics via a non-endo-lysosomal route. Theranostics 8, 3474-3489. doi: 10.7150/thno.23804

Yu, W., Shevtsov, M., Chen, X., and Gao, H. (2020). Advances in aggregatable nanoparticles for tumor-targeted drug delivery. Chin. Chem. Lett. 31, 1366-1374. doi: 10.1016/j.cclet.2020.02.036

Zhang, X., Tian, H., Wu, C., Ye, Q., Jiang, X., Chen, L., et al. (2009). Effect of baicalin on inflammatory mediator levels and microcirculation disturbance in rats with severe acute pancreatitis. Pancreas 38, 732-738. doi: 10.1097/MPA.0b013e3181ad9735

Zhao, Z., Ukidve, A., Krishnan, V., and Mitragotri, S. (2019). Effect of physicochemical and surface properties on in vivo fate of drug nanocarriers. Adv. Drug Deliv. Rev. 143, 3-21. doi: 10.1016/j.addr.2019.01.002

Zhou, X., Hao, Y., Yuan, L., Pradhan, S., Shrestha, K., Pradhan, O., et al. (2018). Nano-formulations for transdermal drug delivery: a review. Chin. Chem. Lett. 29, 1713-1724. doi: 10.1016/j.cclet.2018.10.037

Zhu, W., Jin, Z., Yu, J., Liang, J., Yang, Q., Li, F., et al. (2016). Baicalin ameliorates experimental inflammatory bowel disease through polarization of macrophages to an M2 phenotype. Int. Immunopharmacol. 35, 119-126. doi: 10.1016/j.intimp.2016.03.030

Conflict of Interest: The authors declare that the research was conducted in the absence of any commercial or financial relationships that could be construed as a potential conflict of interest.

Copyright (C) 2020 Zhang, Teng, Li and He. This is an open-access article distributed under the terms of the Creative Commons Attribution License (CC BY). The use, distribution or reproduction in other forums is permitted, provided the original author(s) and the copyright owner(s) are credited and that the original publication in this journal is cited, in accordance with accepted academic practice. No use, distribution or reproduction is permitted which does not comply with these terms. 